\title{
Simultaneous inversion of self-potential for estimating hydraulic parameters and streaming current coefficient
}

\author{
Yusuke OZAKI $^{1,2}$, Hitoshi Mikada ${ }^{1}$, Tada-nori Goto ${ }^{1}$ and Junichi Takekawa ${ }^{1}$ \\ ${ }^{1}$ Dept. of Civil and Earth Res. Eng., Kyoto University \\ ${ }^{2}$ JSPS Research Fellow
}

\begin{abstract}
In this study, we develop a 3D inversion scheme for the transient self potential (SP) data to estimate hydraulic conductivity, streaming current coefficient and specific storage. We test our inversion scheme with the synthetic data according to the pumping test. Our inversion could reconstruct the structure of hydraulic conductivity properly. However, the resolution of the estimation image of specific storage is not as clear as the image of hydraulic conductivity. To detect the factor that decides the resolution in the estimation image of the specific storage, we check the variation of the sensitivity of specific storage during the pumping. The specific storage is sensitive just after the beginning and the termination of the pumping. The stage when the specific storage is sensitive has been kept in the shorter duration than the stage of the hydraulic conductivity. The resolution of estimated image would be affected by the pattern of the transient SP data and pumping data. To improve the resolution in the estimation image of specific storage, the inversion of the synthetic transient SP data according to the repetitive pumping and injection is performed. The inversion image of the specific storage was improved under this condition.
\end{abstract}

\section{INTRODUCTION}

Self-potential (SP) method is useful to detect the groundwater flow because the main source of SP is groundwater flow. The measurements of SP have been performed to detect the subsurface groundwater flow. Recently, several quantitative analysis methods of SP have been proposed, for example, the estimation of elevation of water table (Fournier 1989; Jardani et al., 2009), the estimation of Darcy's velocity (Jardani et al., 2007), the inversion for the estimation of hydraulic conductivity structure (Ozaki et al., in press), etc.

The transient SP profiles are often measured, for example, the SP according to the pumping test (e.g., Rizzo et al., 2004; Jardani et al., 2009). The transient SP data would include more information of the subsurface structures of hydraulic parameters than the SP data under steady condition because transient SP data would reflect the propagation process of the hydraulic pressure according to the change in the pattern of the groundwater flow. The currently proposed analysis methods of the SP data are for the steady state analysis or based on the analytical solution of 1D structure for the transient SP data (Malama et al., 2009). A lot of information in the transient SP data could not be utilized so much and the improvement of the resolution of estimated structure of hydraulic parameters is difficult by these methods. However, the analysis of several data sets of the transient SP data at same time is difficult by the inversion with the ordinal calculation of the sensitivity matrix because the large computational time and memory are required. This difficulty comes from that the inversion of SP is the analysis of the coupled problem of groundwater flow and electrical potential.

In this study, we developed a 3D inversion scheme for the simultaneous estimation of the hydraulic conductivity and the specific storage from transient SP profile. To save the calculation cost for the inversion, the adjoint state method is employed. The streaming current coefficient is also estimated from the estimated hydraulic conductivity and empirical equations. We test our inversion scheme with the synthetic transient SP data. We indicate that the resolution of the estimation image of the specific storage would be controlled by the pattern of the pumping and SP variation.

\section{THEOTY AND METHOD}

\section{Forward calculation}

The governing equation of groundwater flow is described by the diffusive equation as

$$
S \frac{\partial h}{\partial t}=\nabla(K(\nabla h))
$$

, where $S$ is the specific storage $\left[\mathrm{m}^{-1}\right], h$ is the 
hydraulic head, $t$ is the time, $K$ is the hydraulic conductivity $\left[\mathrm{m} \mathrm{s}^{-1}\right]$. The electrical potential $\varphi[\mathrm{V}]$ and hydraulic head are connected by the following equation.

$$
\nabla(\sigma(\nabla \varphi))+\nabla(L(\nabla h))=0
$$

, where $\sigma$ is the electrical conductivity $\left[\mathrm{S} \mathrm{m}^{-1}\right]$ and $L$ is the streaming current coefficient $\left[\mathrm{A} \mathrm{m}^{-2}\right]$. In this study, we ignore the effect of unsaturated zone on the SP. Under this assumption, the hydraulic conductivity and streaming current coefficient are independent on the hydraulic head.

The hydraulic conductivity and streaming current coefficient are known to have the following relationship from the core sample measurements of several kinds of rock or soil

$$
\begin{gathered}
\log \left(Q_{v}\right)=-9.2849-0.8219 \log (k) \\
L=Q_{v} K
\end{gathered}
$$

, where $Q_{v}$ is the electrical charge $\left[\mathrm{C} \mathrm{m}^{-3}\right]$ effective in the generation of SP and $k$ is the permeability $\left[\mathrm{m}^{-2}\right]$ (e.g., Bolève et al., 2007). We calculated the streaming current coefficient from the hydraulic conductivity with equations (3) and (4).

\section{Inverse method}

In the inverse procedure, we used the adjoint state method for the cost-effective calculation of the sensitivity matrix. The SP is the coupled problem of the groundwater flow and electrical potential. The direct calculation of the sensitivity matrices of the hydraulic conductivity and specific storage to the SP, $\partial \varphi / \partial K$ and $\partial \varphi / \partial S$, requires the sensitivity matrices of the hydraulic conductivity and the specific storage to the hydraulic head, $\partial h / \partial K$ and $\partial h / \partial S$, and the sensitivity matrix of hydraulic head to the SP $\partial \varphi / \partial h$. Both the enormous computational time and memory are necessary if this direct calculation of sensitivity matrix is employed. To solve this problem, we used the adjoint state method for the calculation of the sensitivity matrix. We derived the adjoint state equations of equations (1) and (2) according to the calculation process of $\mathrm{Li}$ and Yeh (1998) and Sun and Yeh (1992). The following two adjoint state equations are needed to be solved at same times as the number of observation points.

$$
\begin{gathered}
\delta\left(\boldsymbol{x}-\boldsymbol{x}_{r, i}\right)+\nabla\left(\sigma\left(\nabla w_{2, i}\right)\right)=0 \\
S \frac{\partial w_{2, i}}{\partial t}=-\nabla\left(K\left(\nabla w_{1, i}\right)\right)+\nabla\left(L\left(\nabla w_{2, i}\right)\right)
\end{gathered}
$$

, where $\boldsymbol{x}_{\boldsymbol{i}}$ is the location of the observation point and $w_{1, i}$ and $\mathrm{w}_{2, \mathrm{i}}$ are the solutions of equations (5) and (6). The sensitivity matrices of the hydraulic conductivity $\boldsymbol{J}_{\boldsymbol{h}}$ and the specific storage $\boldsymbol{J}_{\boldsymbol{s}}$ to the SP distribution are

$$
\begin{aligned}
\boldsymbol{J}_{\boldsymbol{h}} & =\frac{\partial \varphi_{i}}{\partial K}\left(t=t_{l}\right) \\
& =\frac{d}{d t_{l}} \int_{t_{0}}^{t_{l}} \int \frac{\partial K}{\partial K} \nabla h \nabla w_{2, i}-\frac{\partial L}{\partial K} f(h) \nabla h \nabla w_{1, i} d V d t \\
\boldsymbol{J}_{\boldsymbol{s}} & =\frac{\partial \varphi_{i}}{\partial S}\left(t=t_{l}\right)=\frac{d}{d t_{l}} \int_{t_{0}}^{t_{l}} \int \frac{\partial S}{\partial S} \frac{\partial h}{\partial t} w_{2, i} d V d t
\end{aligned}
$$

The second term in the right side of equation (7) is the donation from the change of the streaming current coefficient due to the change of the hydraulic conductivity. We define the cost function according to the Tikhonov regularization and minimize it by the hydraulic conductivity and the specific storage. The Gauss-Newton method is employed for the estimation of hydraulic conductivity and specific storage. We estimate the hydraulic conductivity and specific storage simultaneously and then modify the streaming current coefficient from the estimated hydraulic conductivity according to equations of (3) and (4).

\section{SYNTHETIC SP DATA}

In this study, we calculate the SP response with the model of the hydraulic conductivity and the specific storage shown in Figure 1. The uniform structure of the electrical conductivity of $0.025 \mathrm{~S} / \mathrm{m}$ is assigned for the forward calculation. The streaming current coefficient for the forward calculation is calculated from the hydraulic conductivity of the true model with equations (3) and (4). The SP is induced by the pumping at the

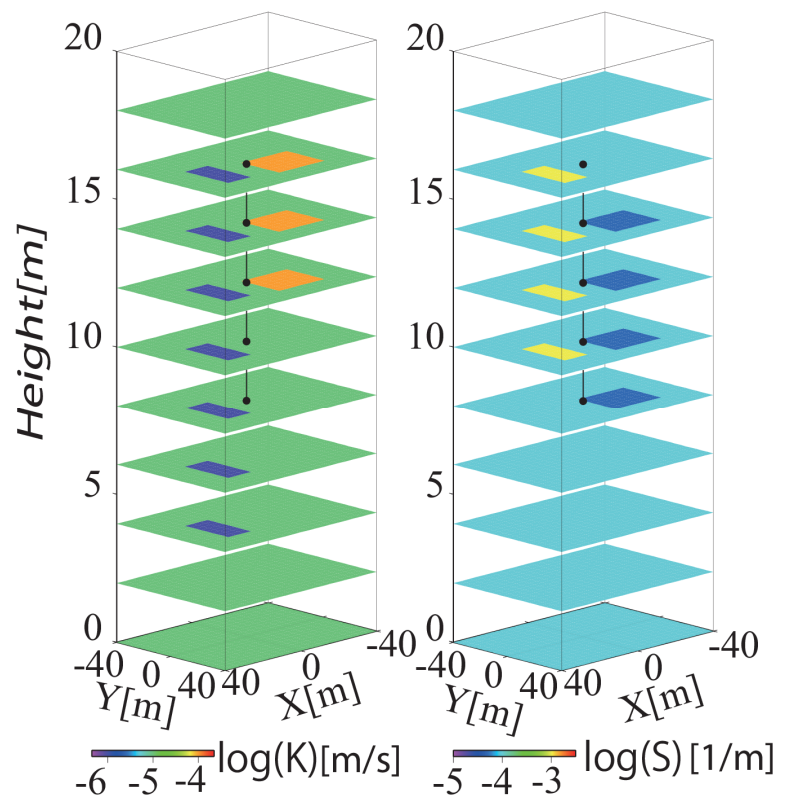

Figure 1 Model of the hydraulic conductivity and the specific storage. The black lines and black dots show the location of the pumping well. 


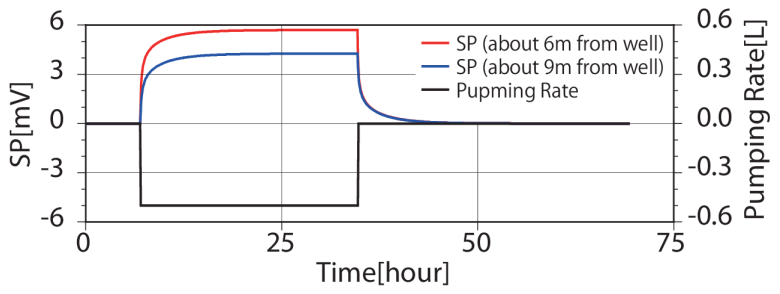

Figure 2 The time variation of the SP at the electrode about $6 \mathrm{~m}$ (red line) and $9 \mathrm{~m}$ (blue line) from the well. The black line shows the time variation of the pumping data.

height from $10 \mathrm{~m}$ to $18 \mathrm{~m}$ in the center of the model. The pumping rate is $0.5 \mathrm{~L} / \mathrm{s}$.

We try to reconstruct the subsurface structure of the hydraulic conductivity and specific storage simultaneously from the synthetic transient SP data. For the inversion test, 36 SP responses, which are measured at about $10 \mathrm{~m}$ intervals on the ground surface, are used as an observed data. The streaming current coefficient is also estimated from the estimated hydraulic conductivity with equations (3) and (4) during inversion. The true structure of the electrical conductivity is given to the inversion as a prior data.

Figure 2 shows the data of pumping and two examples of the transient SP data induced by the pumping for the inversion. The SP increases gradually after the start of pumping. The SP responses become steady state after about 18 hours of the beginning of the pumping and decreased gradually after the termination of the pumping. Such SP data and pumping data are used for the estimation of the hydraulic conductivity and specific storage.

\section{RESULT}

Figure 3 shows the inversion result of the hydraulic conductivity and specific storage from the transient SP profile and pumping data. In the estimation result of hydraulic conductivity, the horizontal high hydraulic conductivity zone and vertical low hydraulic conductivity anomaly can be imaged well. In the estimation result of the specific storage, the vertical high specific storage can be estimated well. However, the horizontal low specific storage anomaly is not estimated clearly. The stage when the specific storage becomes sensitive to the SP is just after the beginning and the termination of the pumping. The term when the specific storage becomes sensitive is much shorter than the term when the hydraulic conductivity is sensitive. To improve the resolution of estimation image of specific storage, the extension of the stage when the specific storage becomes sensitive, e.g., the change of the pumping pattern would be one of the solutions.

\section{Inversion with the different pumping pattern}

To improve the inversion result of the specific storage, we apply our inversion to the SP according to the pumping pattern shown in Figure 4. The transient SP data according to the repetitive pumping and injection test is analyzed to improve the resolution of the estimated specific storage structure. Under this pumping case, the stages of the growth and the decay in the SP variation just after the beginning and the termination of pumping or injection are longer than the stage under the pumping case shown in Figure 2. In these stages, the specific storage is sensitive. The period when the specific storage becomes sensitive can be extended under such repetitive pumping and injection. The improvement of the estimated result of specific storage would be expected by analyzing these transient SP data shown in Figure 4.

Figure 5 shows the inversion result when the transient SP and pumping data in Figure 4 are analyzed. The inversion result of hydraulic conductivity is not so different from the inversion result in Figure 3. In the estimation image of the specific storage, the estimated vertical high specific storage anomaly is not so different from it in Figure 3. However, the estimated horizontal low specific storage area and the estimated value there become wider and lower than them in Figure 3. The estimated image in this area could be improved

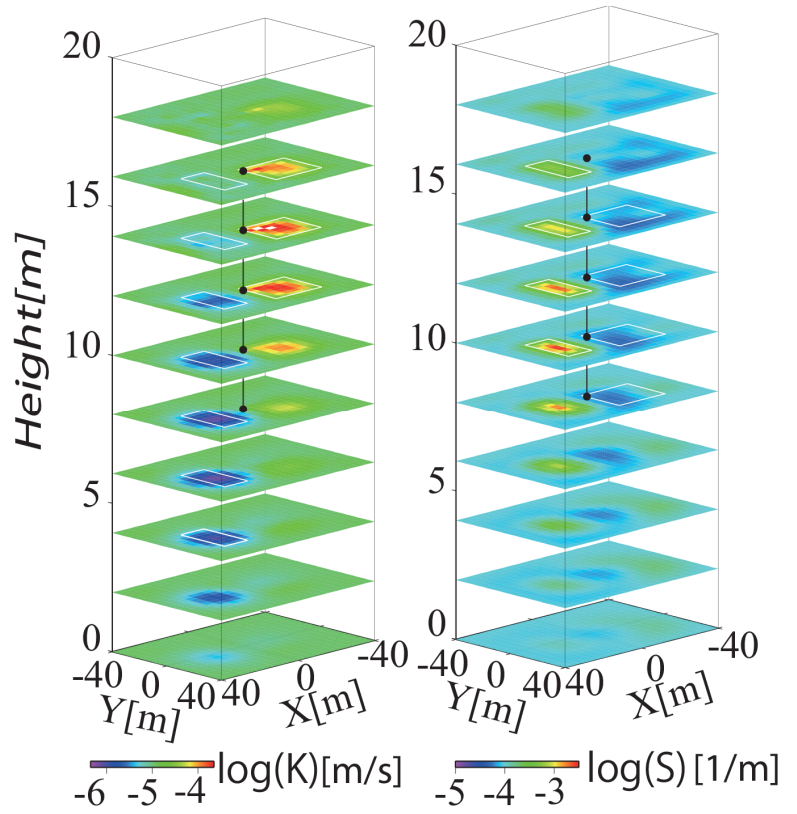

Figure 3 Inversion result of hydraulic conductivity and specific storage from the SP responses and pumping rate shown in Figure 2. 


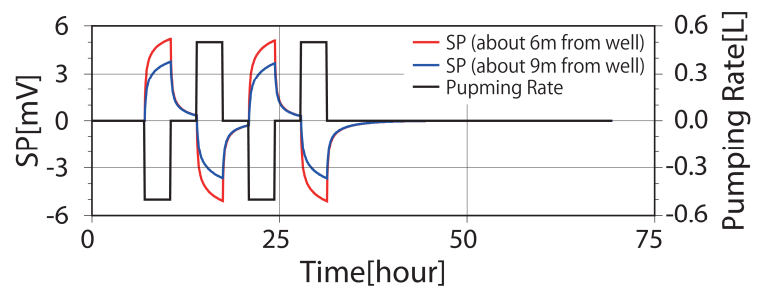

Figure 4 The time variation of the transient SP data at the electrode about $6 \mathrm{~m}$ (Red line) and $9 \mathrm{~m}$ (Blue line) from well. The black line shows the time variation of the pumping data.

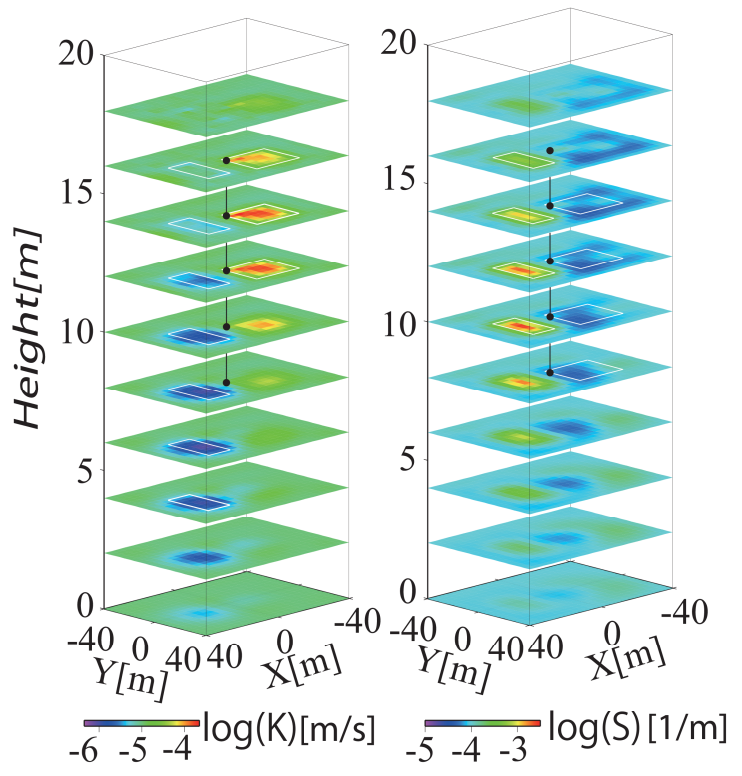

Figure 5 Inversion result of the hydraulic conductivity and the specific storage from the SP responses and pumping data shown in Figure 4.

comparing to the image in Figure 3. From this result, the pumping pattern would be one factor that decides the resolution of the inversion image when the transient SP data is analyzed.

\section{CONLCUTION}

In this study, we develop a 3D inversion scheme for the analysis of transient SP data. The high calculation cost for the sensitivity matrix in the inversion process that comes from the inverse analysis of the coupled problem is saved by the adjoint state method. The structures of both hydraulic conductivity and specific storage structure can be estimated simultaneously by our inversion. From the comparison of the inversion results under different pumping pattern, the control of the resolution of the estimation image would be possible by adjusting the pumping pattern.

ACKNOWLEDGMENT: We are grateful to the Japan Society for the Promotion of Science (JSPS) for the Grants-in-Aid for Science Research in the Research Fellowship program that supported the present study(JSPS: 24.3701).

\section{REFERENCES}

1) Bolève A., A. Revil, F. Janod, J. L. Mattiuzzo and A. Jardani, 2007, Forward Modeling and validation of a new formulation to compute self-potential signals associated with ground water flow: Hydrology and Earth System Sciences, 11, 1661-1671.

2) Fournier, C., 1989, Spontaneous potentials an-d resistivity surveys applied to hydrogeology in a volcanic area: Case history of the Chaîne des Puys (Puy-de-Dôme, France): Geophysical. Prospecting, 37, 647-668, doi: 10.1111/j.1365-2478.1989.tb02228.x.

3) Jardani, A., A. Revil, A. Bolève, A. Crespy, J.-P. Dupont, W. Barrash, and B. Malama, 2007, Tomography of the Darcy velocity from self-potential measurements: Geophysical Research Letters, 34, L24403, doi: 10.1029/2007GL031907.

4) Jardani, A., A. Revil, W. Barrash, A. Crespy, E. Rizzo, S. Straface, M. Cardiff, B. Malama, C. Miller, and T. Johnson, 2009, Reconstruction of the Water Table from Self-Potential Data: A Bayesian Approach: Ground Water, 47, 2, 213-227, doi: 10.1111/j.1745-6584.2008.00513.x.

5) Li, B., and T.-C. J. Yeh, 1998, Sensitivity and moment analysis of head in variably saturated regimes: Advances in Water Resources, 21, 6, 477-485.

6) Malama, B., K. L. Kuhlman, and A. Revil, 2009, Theory of transient streaming potentials associated with axial-symmetric flow in unconfined aquifers: Geophysical Journal International, 179 , 990-1003, doi: 10.1111/j.1365-246X.2009.04336.x.

7) Ozaki Y., H. Mikada, T. Goto and J Takekawa, Self-potential Inversion for The Estimation of Permeability Structure, Journal of Environmental \& Engineering Geophysics, in press.

8) Rizzo, E., B. Suski, A. Revil, S. Straface and S. Troisi, 2004, Self-potential signals associated with pumping tests experiments, Journal of Geophysical Research, 109, B10203, doi: 10.1029/2004JB003049.

9) Sun, N.-Z., and W. W.-G. Yeh, 1992, A stochastic inverse solution for transient groundwater flow: Parameter identification and reliability analysis: Water Resources Research, 28, 12, 3269-3280. 